\title{
Ensayo clinico para la enfermeria basada en evidencia: un desafio alcanzable
}

\author{
Ensaios clínicos para Enfermagem-Baseada-em-Evidências: um desafio possivel \\ Clinical trials for Evidence-Based Nursing: a possible challenge
}

Eugenia Urra Medina ${ }^{1}$, Edith Rivas Riveros ${ }^{2}$, René Mauricio Barría Pailaquilén ${ }^{3}$

\section{RESUMEN}

El ensayo clínico aleatorio constituye el diseño de investigación más riguroso para estudios de intervenciones. En este tipo de estudios, el investigador está interesado en determinar si existe una relación causa-efecto entre un tratamiento y el resultado. Para ello debe comparar grupos de individuos que han sido asignados a recibir diferente nivel de exposición a la intervención, y así determinar si existe el efecto. Hoy en día, se reconoce como uno de los mejores estándares de evidencia para aplicar en la Enfermería Basada en Evidencia. En este contexto, este artículo plantea las principales características de los ensayos clínicos aleatorios, su aplicación, las consideraciones requeridas en su implementación así como sus limitaciones. Con esto se espera estimular su uso para las intervenciones de enfermería en que pueda ser aplicado, hecho relevante ya que desde una perspectiva empírica aportan las mejores pruebas para la Enfermería Basada en Evidencias.

Descriptores: Enfermería basada en la evidencia/métodos Investigación en enfermeira/métodos; Ensayo clínico controlado aleatorio

\section{RESUMO}

O ensaio clínico controlado randomizado é o mais rigoroso método de pesquisa para o estudo de intervenções. Nesses estudos, o pesquisador está interessado em determinar se existe uma relação causal entre a intervenção e o resultado, para o qual compara grupos de indivíduos que tiveram diferente exposição com a intervenção e, assim, avaliar se houve ou não efeito. Hoje é reconhecido como um dos melhores padrões de evidência para aplicar a Enfermagem Baseada em Evidências. Neste contexto, este artigo apresenta as principais características dos ensaios clínicos, sua prática, considerações necessárias para sua execução, bem como suas limitações. O objetivo é incentivar a sua utilização para as intervenções de enfermagem em que possa ser aplicada. Isso é um fato relevante, uma vez que do ponto de vista empírico, eles provêm as melhores evidências para a enfermagem baseada em evidências.

Descritores: Enfermagem baseada em evidencias/métodos; Pesquisa em Enfermagem/métodos; Ensaio clínico controlado aleatório

\begin{abstract}
The randomized controlled clinical trial is the most rigorous research method for studying interventions. In these studies, the researcher is interested in determining whether a causal relationship exists between intervention(s) and outcome(s). This is determined by comparing groups of individuals with different exposure to the intervention, and thus determining the effectiveness of the intervention, or lack thereof. Today this is recognized as one of the best levels of evidence related to Evidence-Based Nursing. In this context, this article presents the main characteristics of clinical trials, their conduct, considerations necessary for implementation, and their limitations. The goal is to encourage the use of clinical trials for applying nursing interventions. This is especially relevant, given that from the empirical point of view, they provide the best basis for evidence based nursing.

Keywords: Evidence-based nursing/methods; Nursing research/methods; Randomized controlled trial

\footnotetext{
${ }^{1}$ PhD. Académica Departamento Enfermería, Universidad de la Serena, La Serena, Chile

${ }^{2}$ Enfermera, MPH. Académica Departamento de Pediatría y Cirugia Infantil, Universidad de La Frontera, Temuco, Chile.

${ }^{3}$ MSc. Académico, Instituto de Enfermería, Universidad Austral de Chile. Valdivia, Chile. Director Oficina de Salud Basada en Evidencia, Facultad de Medicina, Universidad Austral de Chile.
} 


\section{INTRODUCCION}

En el ámbito de salud, el ensayo clínico controlado aleatorio (ECA) es considerado el mejor diseño para evaluar y comparar la efectividad de tratamientos ${ }^{(1-3)}$. Asumiendo que posee los mejores estándares científicos y el más riguroso método para evaluar un tratamiento clínico innovador, el ECA proporciona la más fuerte evidencia científica para tomar una decisión ${ }^{(4)}$, basado en su rigurosidad para determinar si existe una relación de causalidad entre un tratamiento y los resultados; adicionalmente, sirve para proveer la evidencia sobre su costo-efectividad $^{(5)}$. Por este motivo, cada vez más ha sido usado para evaluar intervenciones médicas y quirúrgicas, como también en enfermería y profesiones afines, siendo actualmente incorporados también en educación y las ciencias sociales $^{(\sigma)}$. En los últimos años, ha existido un incremento en el desarrollo de los ECA avalado por su capacidad de establecer con precisión cuáles de los tratamientos que se están realizando están siendo efectivos ${ }^{(3)}$.

De esta forma, el ECA constituye una importante herramienta de investigación para evaluar nuevas intervenciones en salud y favorecer su diseminación. En términos conceptuales, el ensayo clínico se refiere a cualquier experimento que involucre a pacientes y cuyo diseño sirve para elucidar cuál es el tratamiento más apropiado. En general, los ensayos clínicos han llegado a ser sinónimos de ensayo clínico controlado al azar, ya que para que un ensayo clínico logre los estándares de rigurosidad y validez debe cumplir con los requisitos de: ser al azar, ser prospectivo, probar una intervención, incluir uno o más grupos control y comparar las mediciones obtenidas entre los grupos ${ }^{(7)}$.

Su valoración ha sido tan alta que algunos exponen que sólo los ECA permiten hacer inferencias válidas de causa y efecto, teniendo el potencial de influir directamente en el cuidado de los pacientes ${ }^{(8)}$. Los ECA se pueden clasificar en preventivos, que examinan la eficacia de un tratamiento específico en reducir los riesgos asociados a una enfermedad, y terapéuticos, que examinan los resultados de intervenciones específicas o tratamientos tales como el alivio de síntomas o la reducción de riesgos ${ }^{(9)}$.

De este modo, hay variadas razones para conducir un ECA, tales como: evaluar la posibilidad de un nuevo tratamiento para un fin determinado; determinar el uso óptimo de un régimen o procedimiento para lograr un efecto terapéutico; evaluar la eficacia comparativa frente a tratamientos estándares; demostrar un rango de eficacia en la practica habitual; y demostrar la seguridad de una intervención. Otras utilidades consideran la evaluación de pruebas diagnósticas y de intervenciones poblacionales a través de programas sanitarios ${ }^{(10)}$.

Con el objetivo de difundir el ECA como una alternativa de investigación para la práctica enfermera, este artículo revisa sus principales características para su diseño e implementación, así como sus limitantes y dilemas inherentes. Con esto se espera estimular su uso para las intervenciones de enfermería en que pueda ser aplicado, hecho relevante ya que desde una perspectiva empírica aportan las mejores pruebas para la Enfermería Basada en Evidencias (EBE).

\section{ENSAYO CLÍNICO: ASPECTOS CONCEPTUALES}

El ECA es un tipo especial de experimento, que investiga la efectividad de una intervención terapéutica con pacientes, por lo cual se requiere conocer rigurosamente la naturaleza y los alcances de los diseños experimentales. El ECA ha sido definido como un experimento controlado en voluntarios humanos utilizado para evaluar la seguridad y eficacia de tratamientos o intervenciones contra enfermedades y problemas de salud de cualquier tipo ${ }^{(11)}$. Estos experimentos intentan probar si existe una relación causal entre una intervención y un resultado de interés, siendo reconocidos por su capacidad de controlar los sesgos. El control es un proceso sistemático de los diseños experimentales que aísla el efecto de la intervención en estudio al excluir otras causas potenciales de los efectos. Estas otras causas, también llamadas "rivales", se refieren a variables de confusión que pueden proporcionar una explicación alternativa de los efectos sobre la variable dependiente ${ }^{(12)}$. De esta manera, en este diseño experimental, el investigador es un agente activo que usa el razonamiento deductivo para probar o falsear hipótesis, manipulando la variable dependiente mientras intenta mantener otras variables ajenas constantes ${ }^{(2)}$.

Los ECA pueden ser clasificados de distinta manera según criterios específicos. Por fase de investigación si es un ensayo farmacológico (Fase I, II, III y IV), por diseño (paralelo, cruzado o factorial), por su finalidad (estudios de superioridad y equivalencia) y por su aplicabilidad, en pragmáticos y explicativos ${ }^{(10)}$.

Los ECA explicativos se centran en identificar algunos principios subyacentes de un tratamiento, en tanto los pragmáticos focalizan en investigar estos principios en el mundo real de la práctica diaria y ver su aplicabilidad ${ }^{(3)}$. En consecuencia, los ensayos explicativos estudian el efecto de la intervención en las condiciones más experimentales posibles definiendo estrictos criterios de inclusión, mientras los pragmáticos se desarrollan en un escenario más real considerando efectividad, costo y aceptación, estableciendo criterios de selección más amplios y por ende más ajustados a la práctica habitual. Consecuentemente, los explicativos dan cuenta de la eficacia de la intervención, mientras los pragmáticos de su efectividad ${ }^{(3,10)}$.

Las características de un ECA incluyen: asignación aleatoria a los grupos de intervención; desconocimiento de los pacientes e investigadores sobre el tratamiento que fue dado hasta que el estudio se haya completado (no 
obstante, esta condición de "doble ciego" no siempre es factible o apropiada); todos los grupos de estudio son tratados idénticamente, excepto por el tratamiento experimental; los pacientes son generalmente analizados dentro del grupo al cual fueron asignados, independiente de si ellos recibieron la intervención respectiva (análisis por intención de tratamiento); y, el análisis se focaliza sobre la estimación del tamaño de la diferencia en los resultados predefinidos entre los grupos ${ }^{(5)}$. Adicionalmente, un ECA puede ser evaluado en base al rigor como desarrolla las tres propiedades características del diseño: la manipulación, la aleatorización y el control ${ }^{(13)}$.

\section{Manipulación}

A diferencia de los estudios no experimentales que sólo observan los factores de interés y sus desenlaces, los ECA se caracterizan por manipular la variable independiente, es decir, que un investigador hace algo (intervención) cuyo efecto es estudiado. Esta variable independiente corresponde a algún tipo de intervención en salud sea terapéutica, diagnóstica o preventiva. La manipulación consciente y deliberada de la variable independiente es el primer requerimiento de los ECA y permite controlar los sesgos al reducir la probabilidad de que alguna otra condición sea responsable de generar diferencias en las respuestas observadas. En estas condiciones el grupo de pacientes o personas que recibe la intervención en estudio se denomina grupo experimental, de tratamiento o de intervención. Para contrastar el efecto de la intervención en estudio se hace necesario compararlo con otro grupo de personas que no la reciben o reciben un tratamiento o intervención alternativa, por lo general, lo que está disponible en la actualidad, este es el llamado grupo control.

\section{Aleatorización}

Consiste en asignar al azar los participantes a los grupos de estudio, es decir al grupo experimental y al grupo control. Significa que cada paciente tiene una probabilidad conocida, generalmente una chance igual, de ser asignado a cada grupo de tratamiento. Su propósito es crear grupos que sean similares en todos los aspectos excepto en la exposición al tratamiento o intervención. A través de la asignación aleatoria a los grupos de estudio, los factores conocidos y desconocidos que podrían influir en el resultado estudiado son distribuidos equitativamente entre ellos. De esta forma, a través del azar, los dos grupos (experimental y control) serán lo más similar posible, sólo diferenciándose por la exposición a los diferentes tratamientos. El éxito de la aleatorización, por lo tanto, depende de dos procesos interrelacionados. El primero supone generar una secuencia por la cual los participantes son asignados a los grupos de intervención y el segundo, implica la asignación oculta de esta secuencia. No obstante, aunque la aleatorización es el método preferido para equiparar a los grupos y distribuir similarmente las variables de interés, no hay garantía de que los grupos serán efectivamente iguales ${ }^{(10,13)}$.

Un punto fundamental que debe ser considerado es que para poder asegurar la eficacia del método se requiere que la asignación aleatoria sea enmascarada u oculta. Aquí es importante que la persona que asigna los tratamientos no sea la misma que determina la elegibilidad o incorporación de los pacientes, o que el mecanismo de asignación sea hecho por personas externas no involucradas en el ensayo ${ }^{(14)}$. Hay que hacer notar que la asignación oculta es diferente al enmascaramiento o ciego del tratamiento o intervención recibida por los pacientes o participantes del ECA. En este caso, para enfrentar las posibles consecuencias de conocer el tratamiento o intervención que reciben los participantes, su asignación permanece oculta. Estas consecuencias pueden estar determinadas por el conocimiento que tenga el participante, el investigador o quien evalúa los resultados de interés. Para los participantes, el saber lo que están recibiendo puede afectar su respuesta física o psicológica a la intervención. Estos generalmente asumen que los nuevos tratamientos son mejores que el tratamiento estándar, o podría condicionar su cooperación al estudio. En los investigadores, el hecho de conocer la intervención administrada podría determinar hacer evidente sus inclinaciones a favor o contra alguna intervención traspasando esta conducta a los participantes. Además, podrían dar tratamientos o cuidados adicionales a favor de la intervención de interés. Por último, para quienes evalúan los resultados, el conocer la asignación de los tratamientos podría generar una evaluación sesgada de la respuesta ${ }^{(15)}$.

Consecuentemente, para hacer frente a los potenciales sesgos derivados del conocimiento de la intervención administrada, los participantes, los involucrados en su manejo o aquellos que recolectan y analizan los datos clínicos deberían desconocer los tratamientos asignados a los grupos ${ }^{(16)}$. En este escenario pueden darse tres condiciones: simple ciego, cuando los participantes ignoran cual es el tratamiento que están recibiendo (control o de intervención); doble ciego cuando ni el participante ni el recolector de datos sabe cuales sujetos están asignados al grupo tratamiento y control; y triple ciego, cuando ni los participantes del estudio, ni los profesionales entrenados, o el personal que observa o registra los resultados saben la distribución de la asignación a los tratamientos ${ }^{(15-19)}$. No obstante, hay situaciones en las cuales no es posible mantener la intervención oculta, en estas condiciones se habla de un ensayo abierto.

\section{Control}

El poder y fortaleza del diseño experimental se relaciona con el control, el cual puede ser ejercido en varias formas o momentos: muestreo aleatorio, criterios de inclusión y 
exclusión, comparación de los grupos, manipulación de la variable independiente, procedimiento de enmascaramiento y herramientas de análisis estadístico ${ }^{(2)}$. En los experimentos, generalmente uno de los grupos recibe la intervención experimental, mientras otros reciben la intervención estándar o habitual ${ }^{(3)}$. El investigador introduce el control sobre la situación experimental incluyendo, concebir una buena aproximación a un grupo contrafactual, en este caso, el grupo control. El concepto contrafactual, se refiere a lo que hubiera sucedido en la misma población expuesta al factor causal si simultáneamente no lo hubiera recibido. Obviamente es un ideal que no puede ser alcanzado, de tal forma, el grupo control es la mejor aproximación a este ideal $^{(13)}$.

Una representación gráfica de un ECA convencional se muestra en la Figura 1.

\section{ASPECTOS METODOLÓGICOS Y CON- TROVERSIALES DE LOS ECA}

$\mathrm{Al}$ diseñar un ECA debe asegurarse el cumplimiento de sus elementos básicos como forma de incrementar su validez. El rigor científico y estadístico del ECA maximiza la validez interna e incrementa la probabilidad de generalizar sus hallazgos (validez externa) ${ }^{(2)}$. El concepto de validez interna en el ECA se refiere a la magnitud en que las diferencias encontradas entre los grupos aleatorizados son resultado de la intervención evaluada, en tanto la validez externa, se refiere a la magnitud en que los resultados del estudio pueden aplicarse a otros individuos o escenarios ${ }^{(20)}$.

\section{Muestreo}

La técnica de muestreo subyace al establecimiento de criterios explícitos establecidos previo al estudio, exigiéndose seleccionar una muestra aleatoria desde la población de referencia para incorporar a los participantes a diferentes grupos de acuerdo con la aleatorización predefinida ${ }^{(2)}$. Como se ha comentado, la aleatorización es un requisito fundamental del ECA que intenta en primer lugar, una distribución similar de las variables de interés, con el deseo de controlar los factores de confusión. Se considera que la aleatorización es el único método por el cual se puede asegurar que factores desconocidos, potencialmente confundentes pueden ser distribuidos similarmente ${ }^{(21)}$, no obstante esto puede no ocurrir afectando la validez interna.

\section{Reclutamiento}

El éxito del muestreo aleatorio depende del tamaño de la muestra, lo cual suele ser una dificultad cuando el estudio se desarrolla en un solo lugar y por ello no se cuenta con pacientes disponibles. Una forma de sobrellevar el problema es realizar el estudio en diferentes lugares bajo un mismo protocolo, lo que se conoce como estudio multicéntrico. Obviamente esta decisión implica aumentar los costos y requiere de una organización tal que asegure que en todos los lugares el

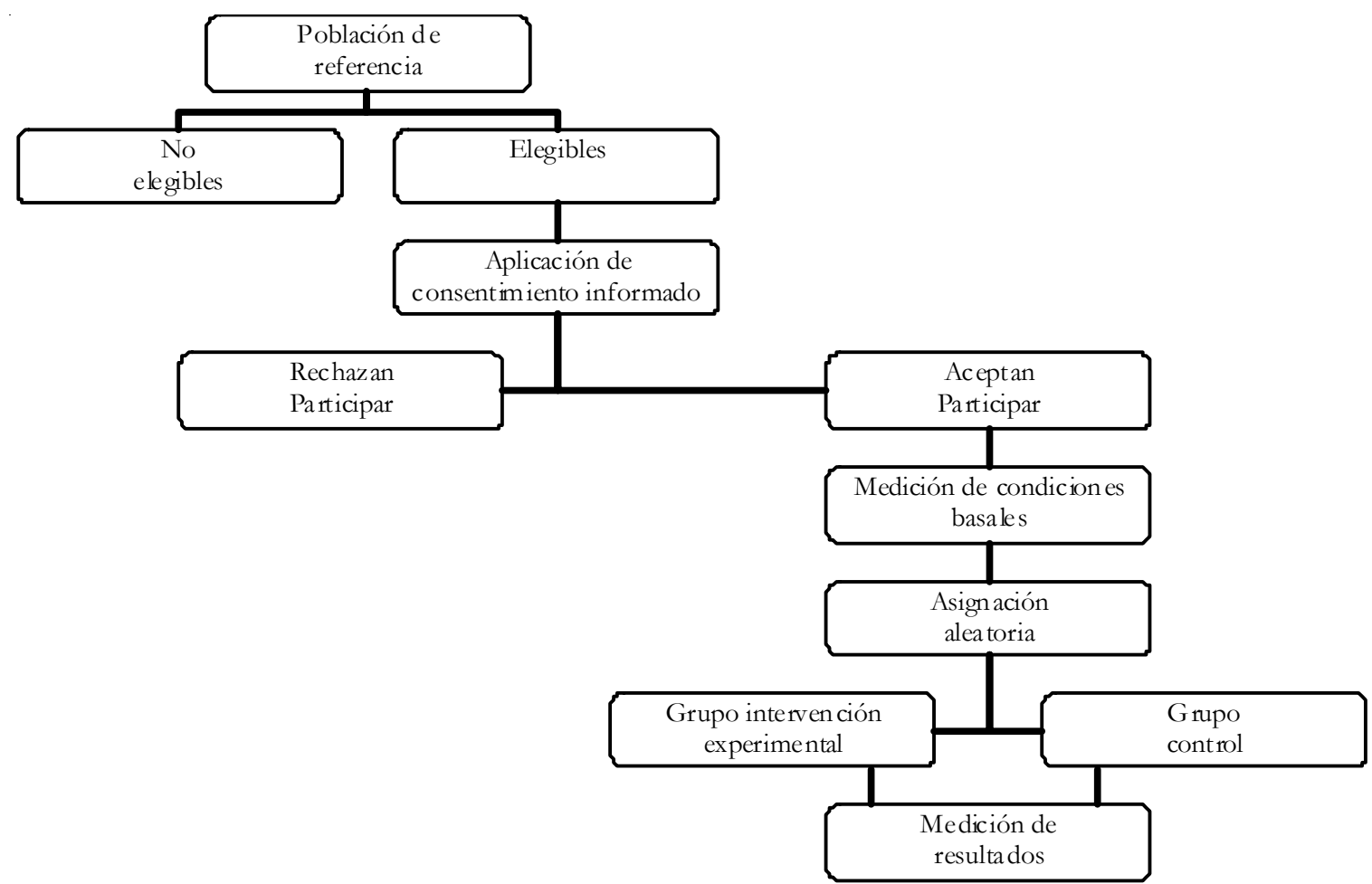

Figura 1 - Diseño de un ECA 
estudio sea conducido de manera similar.

\section{Seguimiento}

Durante la permanencia de los participantes en el estudio, se espera que estos se ciñan al protocolo y reciban efectivamente la intervención asignada aleatoriamente al comienzo. Además, se requiere óptimamente que todos los pacientes que iniciaron el estudio en cada grupo lo culminen como está planificado.

Sin embargo, algunos problemas ocurren que hacen que por un lado abandonen el tratamiento o intervención e incluso reciban la administrada a otro grupo; o bien, abandonan el estudio. En esta situación, frecuente en la vida real, es que se privilegia el uso de ensayos pragmáticos.

\section{RESULTADOS}

Un aspecto importante necesario para interpretar los resultados de un ECA es saber si el tamaño del efecto medido es clínicamente importante. Para ello, hay tres medidas de efecto comúnmente usadas para reportar los resultados: reducción del riesgo relativo, reducción del riesgo absoluto y el número necesario a tratar (NNT) para prevenir un resultado o efecto ${ }^{(12)}$. El NNT es útil porque provee una perspectiva de cómo personas pueden ser beneficiadas en un nivel significativo por la aplicación de un programa o intervención comparado con los que no lo reciben. Adicionalmente, el beneficio esperado puede ser considerado en términos de costo y del riesgo de usar una intervención.

Por último, para la factibilidad de realizar los ECA se debe considerar la capacidad y estructura de la organización (sea hospitalaria o comunitaria) y por cierto, los costos involucrados en la implementación del estudio.

Como ha quedado expuesto, la calidad de los ECA depende de cómo son enfrentados los problemas metodológicos derivados del reclutamiento, la permanencia de los participantes, la adherencia de los sujetos a los tratamientos asignados, el tamaño de la muestra, los lugares involucrados y también los elevados costos que son generalmente cubiertos por fondos de investigación o proyectos.

\section{Cuestionamientos}

Como ya se ha mencionado, la principal fortaleza del ECA está en su poder para establecer causalidad con un diseño rígidamente establecido. Esta aproximación puede no ser lo apropiado para estudios en intervenciones de salud que son difícilmente manejadas en condición de doble enmascaramiento, como las intervenciones o cuidados. En enfermería por lo general se estudia el efecto de múltiples factores o variables y no sólo una relación causal. Sustentado en los principios del experimento cuyo objetivo es explicar el fenómeno a través del método científico/empírico, es criticado desde el ámbito filosófico/epistemológico donde se situé el investigador ya que se asume que los ECA representan al paradigma positivista en salud, que niega la importancia de la experiencia de los individuos o el impacto de las circunstancias individuales y sus relaciones. Similarmente ha sido fuertemente cuestionado como pilar de la $\mathrm{EBE}$ ya que en la visión de algunos, atenta contra la diversidad ${ }^{(22)}$. Se critica además, que dado que las enfermeras no "tratan" pacientes, entonces la búsqueda de asociación y causalidad en los ECA no jugaría un rol vital o preponderante para la investigación en enfermería ${ }^{(21)}$.

También el ECA se enfrenta a problemas de índole ético, por lo que es de suma importancia velar por el cumplimiento de conductas éticas para proteger los derechos y el bienestar de los pacientes y participantes. En este sentido es primordial la aplicación genuina de los consentimientos informados, la evaluación por parte de comités de ética institucionales y el apego a los estándares de las buenas prácticas clínicas ${ }^{(11)}$. Conforme a lo anterior, es necesario evaluar si se justifica el experimento con sujetos humanos, si es posible conducir el estudio acorde a los beneficios posibles y los riesgos potenciales y si se respeta la autonomía de las personas a través de los consentimientos informados, en especial, cuando los sujetos tienen capacidades disminuidas, dificultades de lectura o comprensión o son niños, todas situaciones de vulnerabilidad en el ejercicio de la autonomía. Un aspecto controversial y ampliamente discutido, es el uso de placebos en los grupos de control como es el caso de los ensayos farmacológicos ${ }^{(23-24)}$. Placebo puede ser definido como una sustancia o agente que carece de acción terapéutica y que se utiliza como un control para evaluar la eficacia de un tratamiento o intervención terapéutica. El uso de placebo en los ensayos clínicos es cuestionable ya que se esperaría que la comparación fuera hecha contra la mejor intervención o tratamiento disponible. No obstante, la declaración de Helsinki de la Asociación Médica Mundial establece las condiciones en las cuales el uso de placebo, aún en presencia de tratamientos disponibles, podría ser aceptado ${ }^{(25)}$, enmienda que ha generado controversia y preocupación por los vacíos que deja en su interpretación y con ello, el riesgo de que algunos pacientes sean sometidos a uso de placebo de manera injustificada ${ }^{(26)}$.

Otro punto relevante debe ser la evaluación estricta de la relación riesgo/beneficio, considerándose el concepto clínico de equiponderación (equipoise), esto es, que no haya evidencia que muestre que cualquiera de las intervenciones o tratamientos es mejor que la otra. El investigador verdaderamente desconoce o tiene la incerteza de que la intervención a estudiar no es superior a la existente, ya que de ser así, no sería ético no ofrecerla a todos. En consecuencia, la ética de los ECA requiere de una evaluación cuidadosa de los riesgos y beneficios potenciales y de una 
clara información a los participantes durante el proceso de obtener el consentimiento informado ${ }^{(2)}$.

Para enfermería, el desarrollo de ECA puede generar tensión o conflicto entre las obligaciones con el estudio y las obligaciones o deberes con el paciente. Esto, dependiendo del rol que se asuma en el estudio, vale decir, como investigadora principal, coordinadora o recolectora de datos. Por ejemplo, puede ocurrir que una simple acción de enfermería mejore el efecto del placebo y consecuentemente atenúe las diferencias terapéuticas ${ }^{(27)}$. Entonces, se necesita debate o grupos de ética fuertemente establecidos en las unidades donde se realizan ECA.

\section{APLICACIÓN Y USO DE LOS ECA EN ENFERMERIA}

En enfermería los ECA son aún insuficientes, pero han ido aumentando a medida que las enfermeras y enfermeros adquieren los conocimientos necesarios. Para ejemplificar la variedad de intervenciones que pueden ser estudiadas con un diseño experimental en enfermería se ejemplifican algunos recientes ECA publicados: intervención de empoderamiento en salud ${ }^{(28)}$, contacto piel a piel posterior a parto vaginal ${ }^{(29)}$, efecto del Reiki para la ansiedad previo a colonoscopia ${ }^{(30)}$. En Sudamérica hay algunos ECA que se han realizado en intervenciones como cateterismo vascular en neonatos ${ }^{(31)}$, alternativas de apósitos y duración de accesos venosos ${ }^{(32)}$ y métodos de aspiración en pacientes en ventilación mecánica ${ }^{(33)}$, entre otros.

Los ECA deben responder a tres estándares de calidad: un buen diseño de investigación con la descripción clara y precisa de todas sus partes y pertinencia, un enfoque certero de la validez para asegurar la generalización de los hallazgos (control de la validez interna), y determinar la utilidad de los resultados tanto en la forma de significancia estadística como clínica $^{(12)}$. Con el afán de homogenizar el reporte de los ECA y asegurar que se cumplan sus aspectos fundamentales evitando información incompleta o inexacta, se dispone de recomendaciones para su elaboración: la declaración CONSORT. Revisada con frecuencia, actualmente incluye un listado de 25 ítems que detalla secuencialmente toda la información requerida para elaborar la publicación de un $\mathrm{ECA}^{(34)}$.

Existen tres preguntas claves para una evaluación rápida de un ECA y su eventual implementación en la práctica como aplicación de la $\mathrm{EBE}^{(1,35)}$ :

1. ¿Son válidos los hallazgos del estudio? Para responder esta pregunta hay que analizar los siguientes aspectos: evaluar si hubo una asignación aleatoria a los grupos y si esta asignación fue oculta; establecer si el seguimiento utilizado para evaluar la respuesta o resultado de interés fue suficiente y si todos completaron este seguimiento; evaluar si los pacientes fueron analizados en los grupos a los que fueron originalmente asignados; conocer si el grupo control fue el apropiado; analizar la condición de enmascaramiento (ciego); evaluar si los instrumentos de medición fueron válidos y confiables; por último, establecer si los sujetos asignados a cada grupo fueron similares respecto de sus características basales o de entrada. 2. ¿Cuáles son los resultados del estudio y son ellos importantes? Esta pregunta se refiere a examinar las medidas de resultados y la magnitud de los efectos (por ejemplo, el valor del NNT). Adicionalmente se debe considerar cuán preciso fue este efecto reportado, a través de los intervalos de confianza. Mientras más estrechos los intervalos de confianza, más precisa es la medición del resultado.

3. ¿Estos resultados me ayudarán en el cuidado de mis pacientes? En este caso hay que determinar cuan aplicables son los resultados a los pacientes, cuáles son los riesgos y beneficios y por último, discriminar si la intervención o tratamiento es factible de implementar en el lugar de trabajo.

La valoración crítica es un paso esencial en la toma de decisiones de la EBE. Es indispensable leer y evaluar los estudios en forma regular para facilitar la rapidez con que los profesionales de enfermería pueden juzgar los ECA dentro de una valoración crítica y trasladan los hallazgos aplicables en el lugar de su práctica.

\section{CONCLUSIONES}

Como ha quedado expuesto, el ECA es un diseño de investigación centrado en evaluar intervenciones en el ámbito sanitario, y como tal, es una alternativa disponible para establecer qué intervenciones para el cuidado son las más efectivas en la práctica de enfermería. Como todo tipo de investigación, el ECA posee fortalezas y debilidades, así como sectores de aplicación específicos. A pesar de que es un diseño complejo que requiere de entrenamiento para implementarlo adecuadamente, debería ser considerado por los profesionales de enfermería como alternativa para evaluar las intervenciones que utilizan en su quehacer. En este sentido, para fortalecer las habilidades y competencias en esta área, se requiere que la formación de pregrado incorpore al menos elementos básicos del diseño, los cuales deberían ser desarrollados más ampliamente en ofertas de programas de educación continua y posgrado. Mientras tanto, un avance en el entendimiento actualizado de su metodología y del alcance de sus hallazgos puede ser logrado en base a la lectura crítica de ECA publicados, en grupos de la práctica como de la academia que sistemáticamente puedan hacer revisiones de ella, y su posibilidad de aplicar situaciones o guías en la práctica como lo expone la EBE. 


\section{REFERÊNCIAS}

1. Melnyk BM, Fineout-Overholt E. Rapid critical appraisal of randomized controlled trials (RCTs): an essential skill for evidence-based practice (EBP). Pediatr Nurs. 2005;31(1):50-2.

2. Walker W. The strengths and weaknesses of research designs involving quantitative measures. J Res Nurs. 2005;10(5):571-82.

3. Newell R, Burnard P. Randomised controlled trials. In: Newell R, Burnard P, editors. Vital notes for nurses: research for evidence-based practice. New Jersey: WilleyBlackwell; 2006. p. 163-71.

4. Watson B, Procter S, Cochrana W. Using randomised controlled trials (RCTs) to test service interventions: issues of standardisation, selection and generalisability. Nurse Res. 2004;11(3):28-42.

5. Sibbald B, Roland M. Understanding controlled trials. Why are randomised controlled trials important? BMJ. 1998;316(7126):201.

6. Boruch R, Rui N. From randomized controlled trials to evidence grading schemes: current state of evidence-based practice in social sciences. J Evid Based Med. 2008;1(1):41-9.

7. Fetter MS, Feetham SL, D'Apolito K, Chaze BA, Fink A, Frink BB, et al. Randomized clinical trials: issues for researchers. Nurs Res. 1989;38(2):117-20.

8. Altman DG. Better reporting of randomised controlled trials: the CONSORT statement. BMJ. 1996;313(7057):5701. Comment in: BMJ. 1997;314(7087):1127. BMJ. 1997;314(7087):1126-7. BMJ. 1997;314(7087):1126; author reply 1127.

9. Lengacher CA, Gonzalez LL, Giuliano R, Bennett MP, Cox CE, Reintgen DS. The process of clinical trials: a model for successful clinical trial participation. Oncol Nurs Forum. 2001;28(7):1115-20.

10. Irala J, Martínez MA, Seguí M, Jiménez JJ. Diseños de investigación en epidemiología. In: Irala Estévez J, Martínez-González MA, Seguí-Gómez M. Epidemiología aplicada. 2a ed. Barcelona: Ariel; 2008. p. 214-36.

11. Lazcano-Ponce E, Salazar-Martínez E, GutiérrezCastrellón P, Angeles-Llerenas A, Hernández-Garduño A, Viramontes JL. [Randomized clinical trials: variants, randomization methods, analysis, ethical issues and regulations]. Salud Publica Mex. 2004;46(6):559-84. Spanish.

12. Salmond SS. Randomized controlled trials: methodological concepts and critique. Orthop Nurs. 2008;27(2):116-22; quiz 123-4. Comment on: Orthop Nurs. 2008;27(2):111-5.

13. Polit D, Beck CT. Designing quantitative studies. In: Polit DF, Beck CT, editors. Nursing research: generating and assessing evidence for nursing practice. 8th ed. Philadelphia, PA: Lippincott Williams \& Wilkins; 2008. p. 248-85

14. Altman DG, Schulz KF. Statistics notes: Concealing treatment allocation in randomised trials. BMJ. 2001;323(7310):446-7.

15. Schulz KF, Chalmers I, Altman DG. The landscape and lexicon of blinding in randomized trials. Ann Intern Med. 2002;136(3):254-9.

16. Day SJ, Altman DG. Statistics notes: blinding in clinical trials and other studies. BMJ. 2000;321(7259):504.

17. Conlon M, Anderson GC. Three methods of random assignment: comparison of balance achieved on potentially confounding variables. Nurs Res. 1990;39(6):376-9.

18. Hauck WW, Gilliss CL, Donner A, Gortner S. Randomization by cluster. Nurs Res. 1991;40(6):356-8.

19. Rudy EB, Vaska PL, Daly BJ, Happ MB, Shiao P. Permuted block design for randomization in a nursing clinical trial. Nurs Res. 1993;42(5):287-9.

20. Eldridge S, Ashby D, Bennett C, Wakelin M, Feder G. Internal and external validity of cluster randomised trials: systematic review of recent trials. BMJ. 2008;336(7649):876-80.

21. Richards DA, Hamers JP. RCTs in complex nursing interventions and laboratory experimental studies. Int J Nurs Stud. 2009;46(4):588-92. Comment in: Int J Nurs Stud. 2009;46(8):1156-8; author reply 1159-60.

22. Rolfe G. Faking a difference: evidence-based nursing and the illusion of diversity. Nurse Educ Today. 2002;22(1):312; discussion 13-4.

23. Rothman KJ, Michels KB. The continuing unethical use of placebo controls. N Engl J Med. 1994;331(6):394-8. Comment in: N Engl J Med. 1995;332(1):62. N Engl J Med. 1995;332(1):61-2. N Engl J Med. 1995;332(1):61; author reply 62. N Engl J Med. 1995;332(1):60; author reply 62. N Engl J Med. 1995;332(1):60-1; author reply 62. N Engl J Med. 1995;332(1):61; author reply 62. N Engl J Med. 1995;332(1):61; author reply 62. N Engl J Med. 1995;332(1):62. N Engl J Med. 1995;332(1):61; author reply 62. N Engl J Med. 1995;332(1):60; author reply 62.

24. Simon R. Are placebo-controlled clinical trials ethical or needed when alternative treatment exists? Ann Intern Med. 2000;133(6):474-5. Erratum in: Ann Intern Med 2000;133(9):754.

25. World Medical Association. Declaration of Helsinki Ethical Principles for Medical Research Involving Human Subjects. [cited 2010 Apr 18]; Available in: http:// www.wma.net/en/30publications/10policies/b3/ 17c.pdf

26. Michels KB, Rothman KJ. Update on unethical use of placebos in randomised trials. Bioethics. 2003;17(2):188204.

27. Oberle K, Allen M. Ethical considerations for nurses in clinical trials. Nurs Ethics. 2006;13(2):180-6.

28. Crawford Shearer NB, Fleury JD, Belyea M. Randomized control trial of the Health Empowerment Intervention: feasibility and impact. Nurs Res. 2010;59(3):203-11.

29. Gouchon S, Gregori D, Picotto A, Patrucco G, Nangeroni M, Di Giulio P. Skin-to-skin contact after cesarean delivery: an experimental study. Nurs Res. 2010;59(2):78-84.

30. Hulse RS, Stuart-Shor EM, Russo J. Endoscopic procedure with a modified Reiki intervention: a pilot study. Gastroenterol Nurs. 2010;33(1):20-6.

31. Barría RM, Lorca P, Muñoz S. Randomized controlled trial of vascular access in newborns in the neonatal intensive care unit. J Obstet Gynecol Neonatal Nurs. 2007;36(5):4506.

32. Machado AF, Pedreira MLG, Chaud MN. Estudo prospectivo, randomizado e controlado sobre o tempo de permanência de cateteres venosos periféricos em crianças, segundo três tipos de curativos. Rev Latinoam Enferm. 2005;13(3):291-8.

33. Zeitoun SS, de Barros AL, Diccini S. A prospective, randomized study of ventilator-associated pneumonia in patients using a closed vs. open suction system. J Clin Nurs. 2003;12(4):484-9.

34. Schulz KF, Altman DG, Moher D; CONSORT Group. CONSORT 2010 Statement: Updated guidelines for reporting parallel group randomised trials. J Clin Epidemiol. 2010;63(8):834-40.

35. Cullum N. Evaluation of studies of treatment or prevention interventions. Evid Based Nurs. 2000;3(4):1002. 